\title{
ON THE LOCATION OF ROOTS OF POLYNOMIALS AND ENTIRE FUNCTIONS*
}

\author{
BY E. B. VAN VLECK
}

On account of its importance, the problem of the location of the roots of a polynomial occupies the central position in the theory of equations. For the most part the attention of mathematicians has been confined to the real roots of real polynomials-i.e., polynomials with real coefficients. So general has been the assumption of the reality of the polynomial that it has often, unfortunately, not been stated in theorems in which it is vital; for example, when it is said that imaginary roots enter in conjugate pairs. Correspondingly, the tools for the investigations were ordinary algebra and the calculus. When, however, one's thought is extended to the location of imaginary roots and to unreal polynomials, the border line is crossed, as the treatment involves almost inevitably considerations and methods belonging to the theory of analytic functions. It is in the overlapping region belonging alike to the theory of equations and that of analytic functions that we will stray today.

After the notable advances of Budan, Fourier, Cauchy, Laguerre and others, investigation of the root-location of polynomials and entire functions was largely neglected for other work. With the new century has come a new activity. It is my pleasure to bring before you today some of the important advances made and some theorems discovered since 1900. This will, I hope, be the more profitable because the new results lie scattered and untied through the periodical literature and have not yet become a part of current texts. $\dagger$ Some of them, though little known today, are destined to become classic.

* An address presented at the invitation of the program committee at a Symposium held at the meeting of the Society in Chicago, March 29, 1929.

† Except Bieberbach's noteworthy Vorlesungen über Algebra, 1928. 
Time limitations force me to omit certain aspects and developments which I had purposed originally to include. Reluctantly I omit inclusion of the line of work* inaugurated by Landau and Montel. The underlying thought in this work is to give $p+1$ coefficients in an equation

$$
P_{n}(z) \equiv a_{0}+a_{1} z+a_{2} z^{2}+\cdots+a_{n} z^{n}=0, \quad\left(a_{0} \neq 0\right),
$$

leaving the other coefficients arbitrary, and to seek the limitations thereby imposed upon the roots. Thus Montel supposes the first $p+1$ consecutive coefficients $a_{0}, \cdots, a_{p}$ to be given in this equation or, more generally, in one of any degree with $n+1$ non-zero terms, and shows that then the $p$ roots of smallest absolute value are conditioned to lie in a circle whose radius depends only on the values of these given coefficients and on $n$. I omit also consideration of special kinds of polynomials, computational methods for calculating the roots, polynomials with a parameter, chains of polynomials arising through continued fractions or other algorithms, extensions of Descartes' Rule of Signs or of the Hermite-Poulain Theorem, etc. I have not sought to give today an encyclopaedic report but rather so to select the material as to secure some unity of theme and thought.

At the end of the report is appended a bibliography which includes also some omitted topics allied to those considered here. Literature references to the bibliography are indicated in the report by bracketed numbers.

\section{PART I}

One succession of modern theorems was inaugurated by the Gauss-Lucas Theorem on the location of the roots of the derivative of any polynomial, real or imaginary. This theorem, though so fundamental, is strangely enough but little known popularly.

Gauss-Lucas Theorem. If in the complex plane of $z=x+i y$ we mark off the convex polygon whose vertices are

* See the bibliography. 
roots of a given polynomial and which contains either within or upon its boundary all other roots of the polynomial, then none of the roots of the derived polynomial will lie exterior to this polygon, and a root will fall upon its boundary only when coincident with a multiple root of the given polynomial on the boundary.

This theorem was definitely stated and proved by Lucas [63] in 1879 and perhaps can be best known by his name, but it follows as an immediate corollary from an observation of Gauss [62] appended as a handschriftliche Bemerkung after a memoir in his Collected Works. Gauss says that the roots of the derivative of any given polynomial are the positions of equilibrium in a plane field of force created by placing unit-masses at the roots of the given polynomial which repel (or just as well attract) with a force inversely proportional to the distance. A mass $r$ is, of course, to be placed at each $r$-fold root. For proof of this Equilibrium Theorem of Gauss* we have merely to observe that the roots of the derivative are given by the equation

(2) $\frac{P_{n}^{\prime}(z)}{P_{n}(z)} \equiv \sum \frac{1}{z-\alpha_{j}} \equiv \sum \frac{1}{r_{j} e^{i \theta_{j}}}=0, \quad(j=1, \cdots, n)$,

where the $\alpha_{j}$ are the roots of the given polynomial and the $r_{j}, \theta_{j}$ denote the lengths and arguments of the vectors drawn from these roots to a point $z$. Then, if each $\theta_{j}$ is changed into $-\theta_{j}$ to convert the terms into their conjugates, each term in (2) becomes a vector representing a force due to a unit mass situated at $\alpha_{j}$ which repels inversely as the distance, and the equation gives the positions of equilibrium, where there is no force. Now it is obvious that if we produce any side of the convex polygon indefinitely and $z$ is a point within the half plane not containing the polygon, the components of force at $z$ perpendicular to the side will all be directed away from the polygon, thereby reinforcing each other so that equilibrium at $z$ is impossible. Like considera-

* Rediscovered by Lucas, Comptes Rendus, vol. 89 (1879), pp. 224-226. 
tions apply when $z$ lies on a side and is not coincident with a root of the given polynomial. The theorem of Lucas thus follows as a corollary.

Beside this theorem of Gauss-Lucas we may now place a beautiful result published without proof by Jensen [72] in 1913, which gives much more definite information regarding the derived polynomial when the given polynomial is real.

Jensen's Theorem. If circles are described having as diameters the segments connecting pairs of conjugate roots of the given polynomial, then no imaginary root of the derivative of the polynomial can lie exterior to all these circles.

The first published proof [73] of this theorem we owe to Walsh (1920-1921); it is likewise based on the above Equilibrium Theorem of Gauss. Walsh sets a repulsive unitmass at each root of the given polynomial and shows that at any point not on the real axis and exterior to the circle having as diameter the segment joining a pair of conjugate imaginary roots $\alpha_{j}, \bar{\alpha}_{j}$, the net force due to the masses at these two points will have a non-vanishing component perpendicular to the real axis and directed away from this axis. The like is obviously true of the repulsive force due to the masses upon the real axis. Hence at any point exterior to all the Jensen circles and not on the real axis these components reinforce one another and equilibrium is impossible. Consequently no imaginary root of the derived polynomial can lie exterior to all the Jensen circles. Walsh apparently did not notice that the introduction of repulsive masses was an unnecessary trapping and that without essential modification the proof could be thrown into the following elementary form [74], quite possibly that of Jensen himself. Let $\alpha=\beta+i \gamma, \bar{\alpha}=\beta-i \gamma$ be a pair of conjugate imaginary roots of $P_{n}(z)$. The corresponding component in (2) is

$$
\begin{aligned}
& \frac{1}{(x+i y)-(\beta+i \gamma)}+\frac{1}{(x+i y)-(\beta-i \gamma)} \\
& =\frac{2(x-\beta)\left[(x-\beta)^{2}+y^{2}+\gamma^{2}\right]-2 i y\left[(x-\beta)^{2}+y^{2}-\gamma^{2}\right]}{\left[(x-\beta)^{2}+(y-\gamma)^{2}\right] \cdot\left[(x-\beta)^{2}+(y+\gamma)^{2}\right]} .
\end{aligned}
$$


The coefficient of $i$ is opposite in sign to $y$ if $(x-\beta)^{2}+y^{2}-\gamma^{2}$ $>0$; that is, when $z$ lies outside the circle $(x-\beta)^{2}+y^{2}=\gamma^{2}$. For a real root $\beta$ the component is

$$
\frac{1}{x+i y-\beta}=\frac{(x-\beta)-i y}{(x-\beta)^{2}+y^{2}},
$$

which also has a sign opposite to that of $y$ for the coefficient of $i$. Hence at any point exterior to all the Jensen circles and not on the real axis the coefficient of $i$ in $P_{n}^{\prime} / P_{n}$ does not vanish. This proves the theorem.

Walsh supplements Jensen's Theorem by showing that in any segment of the real axis which is free from roots of the real polynomial $P_{n}(z)$ and which does not penetrate into any of its Jensen circles there can lie at most only one root of its derivative $P_{n}^{\prime}(z)$. Further, if any Jensen circle has no point in common with any other Jensen circle in part exterior to it and contains exactly $k$ roots of $P_{n}(z)$, then it will contain at least $k-1$ roots of its derivative and at most, $k+1$ roots. I may add that I find that if we amalgamate each series of overlapping circles into a single connected region so as to form Jensen regions exterior to one another, the conclusion still holds with respect to these regions.

These results can be readily established analytically without following the Gaussian suggestion of introducing repulsive masses at the roots of the given polynomial, but that method is undeniably illuminating and suggestive, and has the advantage of being independent of the selection of the coordinate axes. Bôcher [68] applied the method to the Jacobian

$$
\left|\begin{array}{ll}
\frac{\partial \phi}{\partial z_{1}} & \frac{\partial \phi}{\partial z_{2}} \\
\frac{\partial \dot{\psi}}{\partial z_{1}} & \frac{\partial \psi}{\partial z_{2}}
\end{array}\right|
$$

of two binary forms $\phi_{p_{1}}\left(z_{1}, z_{2}\right), \psi_{p_{2}}\left(z_{1}, z_{2}\right)$ of degrees $p_{1}$ and $p_{2}$ respectively. It is easy then to see that the roots $z=z_{1} / z_{2}$ 
of 'the Jacobian, other than multiple roots of either form or common roots of both forms, are the equilibrium positions in a field of force due to unit masses placed at the roots of $\phi$ and of $\psi$ which respectively attract and repel with a force inversely proportional to the distance. Continuing the application, Walsh has made an extended study of the location of the roots of the Jacobian. In a number of memoirs he has also applied the Gaussian idea to derive some nice theorems relating to the roots of the derivative of a polynomial. For example, he shows [71] that if $m_{1}$ roots of a polynomial lie in a circle of radius $r_{1}$ and center $\alpha_{1}$ and all the remaining $m_{2}$ roots lie in a circle of radius $r_{2}$ and center $\alpha_{2}$, all the roots of the derivative lie in these two circles and a third circle whose center is $\left(m_{1} \alpha_{2}+m_{2} \alpha_{1}\right) /\left(m_{1}+m_{2}\right)$ and whose radius is $\left(m_{1} r_{2}+m_{2} r_{1}\right) /\left(m_{1}+m_{2}\right)$. If, furthermore, these circles are all external to one another, then the three circles contain respectively $m_{1}-1, m_{2}-1$, and one root respectively.

I may add that the Gaussian method and various resulting conclusions-for example, Lucas and Jensen's theoremsapply not only to the derivative of the given polynomial $P_{n}(z)$ but also to any polynomial $\psi_{n-1}(z)$ of degree $n-1^{*}$, for which we have a decomposition into partial fractions of the form

$$
\frac{\psi_{n-1}(z)}{P_{n}(z)}=\sum_{j=1}^{n} \frac{A_{j}}{z-\alpha_{j}}
$$

in which the $A_{j}$ are all positive.

The next theorem which I wish to cite relative to the roots of the derivative of a polynomial is due to Grace and arose from an attempt to obtain a generalization of Rolle's Theorem valid for two unreal roots of a polynomial. According to Rolle's Theorem, if a real polynomial vanishes at two different points of the real axis (or has the same value at these points), there will lie at least one root of its derivative between them. Thus two real roots of a real polynomial fixate

* For extension of the Jensen Theorem, the polynomial $\psi_{n-1}(z)$ is, of course, to be taken real as well as $P_{n}(z)$. 
one root of the derivative. In what direction should one look for a corresponding result for imaginary roots and unreal polynomials? Doubtless this question occurred to others before Grace, and we may conjecture that it was on account of its difficulty that no answer was obtained till the present century. In November 1901, in a notable five-page article [75], Grace obtained the following important and strange theorem.

THEOREM OF GRACE. If $a$ and $b$ are any two roots of any polynomial, real or unreal, of degree $n$, then within or on a circle having the center $(a+b) / 2$ and a radius one-half of $|a-b| \operatorname{ctn}(\pi / n)$ there will be at least one root of the derived polynomial.

This can scarcely be called a generalization of Rolle's Theorem inasmuch as it does not reduce to Rolle's Theorem for the special case of a real polynomial with two real roots. Further, in anticipation of the second part of my report today, I may add that it is not a theorem that admits of extension to entire functions, since as $n$ increases indefinitely, the radius of the circle also increases indefinitely. No information could therefore be afforded regarding the derivative of the given entire function except that it had a root somewhere in the complex plane. But this conclusion would be false since the entire function $e^{z}-1$ has a periodic system of zeros, while its derivative is devoid of zeros.

Grace's proof of his theorem was essentially invariantive in character. If in an algebraic equation

$$
P_{n}(z) \equiv a_{0}+a_{1} z+a_{2} z^{2}+\cdots+a_{n} z^{n}=0,
$$

we put $z=x_{1} / x_{2}$, the left-hand member after dropping the denominator is converted into an algebraic form in $x_{1}$ and $x_{2}$. If we take a second equation

$$
b_{0}+b_{1} z+b_{2} z^{2}+\cdots+b_{n} z^{n}=0
$$

the two equations may be said to be apolar when the corresponding bilinear forms are apolar; that is, if their coefficients are connected by the equation 
(4) $a_{0} b_{n}-\frac{1}{C_{1}^{n}} a_{1} b_{n-1}+\frac{1}{C_{2}{ }^{n}} a_{2} b_{n-2}-\cdots+(-1)^{n} a_{n} b_{0}=0$.

Starting from Lucas' Theorem, Grace first establishes between two apolar equations a most important and far reaching relation which I shall term Grace's Apolarity Theorem.

Grace's A polarity Theorem. If two algebraic equations are apolar, then any circle in the z-plane which contains all the roots of either equation will contain at least one root of the other.

The term circle is here to be understood in the sense of the theory of functions and may be indifferently the closed interior or the closed exterior of a circle, or a half plane inclusive of its boundary.

It will be noticed that if any two of the equations (1), (3), (4) are given, we can immediately write down the third. Hence the theorem says that if any algebraic equation (1) is given whose coefficients are connected by a homogeneous linear relation (4), we can immediately write down a third equation (3) such that every circle which contains all the roots of (1) will contain at least one root of (3), and reciprocally.

Grace applies this thorem to the derivative of (1). If we substitute in (1) any two of its roots $\alpha$ and $\beta$ and subtract, we obtain a homogeneous linear relation

$$
a_{1}(\alpha-\beta)+a_{2}\left(\alpha^{2}-\beta^{2}\right)+\cdots+a_{n}\left(\alpha^{n}-\beta^{n}\right)=0
$$

between the coefficients of the derived equation. The equation (3) which corresponds to this linear relation is

$$
\begin{aligned}
\left(\alpha^{n}-\beta^{n}\right)-n\left(\alpha^{n-1}-\beta^{n-1}\right) z & +C_{2}^{n}\left(\alpha^{n-2}-\beta^{n-2}\right) z^{2}+\cdots \\
& +(-1)^{n-1} C_{1}^{n}(\alpha-\beta) z^{n-1}=0,
\end{aligned}
$$

which may be written

$$
(\alpha-z)^{n}-(\beta-z)^{n}=0 .
$$

Hence at least one root of the derivative $P_{n}^{\prime}(z)$ must lie in 
any circle containing all the roots of (5). By extracting the $n$th root in (5) we get for its roots

$$
z=\frac{\beta \omega_{j}-\alpha}{\omega_{j}-1}, \quad(j=1,2, \cdots, n-1)
$$

where $\omega_{j} \equiv \cos (2 j \pi / n)+i \sin (2 j \pi / n)$ denotes an $n$th root of unity. This can be easily reduced to the form

$$
z=\frac{\alpha+\beta}{2}+i \frac{\alpha-\beta}{2} \operatorname{ctn}\left(\frac{j \pi}{n}\right),
$$

which shows that the $n$ roots of (5) all lie on a straight line perpendicular to the segment joining $\alpha$ and $\beta$ at its middle point. The most remote of these roots is at a distance one-half of $|\alpha-\beta| \operatorname{ctn}(\pi / n)$ from this middle point. Consequently a circle described around this mid-point as center and with a radius equal to this distance contains all the roots of the equation (5) apolar to the derivative $P_{n}^{\prime}(z)$ and hence by Grace's apolarity theorem encloses at least one root of $P_{n}^{\prime}(z)$. Thus two roots $\alpha$ and $\beta$ of a given polynomial fixate one root of the derivative in the manner stated.

In 1907 this theorem was re-derived, apparently independently, by Heawood [76]. He noted the fact that for $n>3$ the region in which lies at least one root of the derivative need not be extended equally in every direction from the center $(\alpha+\beta) / 2$ to the perimeter of the circle. When $n=4$, the minimum region is that covered by two circles of radius one-third of $|\alpha-\beta| \operatorname{ctn}(\pi / n)$ and having their centers on the perpendicular to the segment joining $\alpha$ and $\beta$ at its middle point and at a distance one-sixth of $|\alpha-\beta| \operatorname{ctn}(\pi / n)$. For greater values of $n$ the form of the curve bounding the region is very complicated and scarcely specifiable. Heawood established Grace's Theorem by showing that the root of the derivative nearest to the mid-point $(\alpha+\beta) / 2$ attains its maximum distance only when all the roots of the derivative are equal; that is, only when $P_{n}^{\prime}(z) \equiv c(z-\gamma)^{n-1}$, so that we may write $P_{n}(z) \equiv c(z-\gamma)^{n} / n+c_{1}$. Substituting the roots $\alpha$ 
and $\beta$ in turn, we find $\left.(\alpha-\gamma)^{n} \equiv \beta-\gamma\right)^{n}$, and $\gamma$ is consequently a root of equation (5). The theorem then follows as before.

The most interesting successor of Grace's memoir is one published in 1922 by Szegö [77] with the modest title: Bemerkungen zu einem Satz von J. H. Grace ueber die Wurzeln algebraischer Gleichungen. He derives Grace's Theorem on apolar polynomials on the basis of what he calls a Faltungssatz, which I shall call his Coincidence-Theorem. This runs as follows.

Szegö's Coincidence Theorem. If $S_{1}$ denotes the sum of the products of $z_{1}, z_{2}, \cdots, z_{n}$ taken $j$ at a time and these are any $n$ quantities satisfying the equation

$$
a_{0}+a_{1} S_{1}+\cdots+a_{n} S_{n}=0,
$$

then any circle which contains all the roots of the equation

$$
a_{0}+C_{1}{ }^{n} a_{1} z+C_{2}^{n} a_{2} z^{2}+\cdots+a_{n} z^{n}=0
$$

will contain at least one of any of the $n$ quantities satisfying (6).

It will be noticed that equation (7) results from (6) when the $z_{j}$ are all coincident. Szegö's proof, not altogether easy, is by means of mathematical induction. An obvious consequence of his theorem is that if any set of the $z_{j}$ satisfying (6) all lie in (that is, on or within) a circle, not all the roots of (7) lie without. We have thus Walsh's theorem.

THEOREM OF WALSH. If any circle contains a set of values of $z_{1}, \cdots, z_{n}$ satisfying (6), it must contain at least one root of (7).

This result, in a somewhat different formulation, was published almost simultaneously as Theorem I and the "main result" of one of Walsh's principal papers [78] and was established by geometrical considerations involving continuity. It is not, however, to be regarded as a subordinate corollary of Szegö's Theorem, inasmuch as the latter is obviously also an immediate corollary of the former. The relation of the two theorems was not pointed out by Walsh.

The usefulness of the Szegö-Walsh Theorem is revealed 
by very different sets of theorems which the two authors rapidly deduce from it. I have time to mention only one or two of each. As a typical theorem of Walsh, I select the following [78, p. 173]: Let

$$
\left(z-\alpha_{1}\right)\left(z-\alpha_{2}\right) \cdots\left(z-\alpha_{n}\right)-A=0
$$

be an equation with a parameter $A$; then if $\alpha_{1}, \cdots, \alpha_{n}$ are all situated in a circle of radius $r$ and center $\alpha$, the $n$ roots of (8) will all be contained in $n$ circles whose centers $\alpha+A^{1 / n}$ form a regular polygon with center at $\alpha$ and which have the common. radius $r$. If none of these circles overlap, there will be just one root in each circle. The second of his theorems which I wish to cite is a special case of his "Main result" and is set off as his Theorem II.* This says that "if the points $\alpha_{1}, \alpha_{2}, \cdots, \alpha_{k}$ lie in a circular region $C$ and if $z$ is any point exterior to $C$, the root of the equation in $\alpha$

$$
\frac{1}{z-\alpha_{1}}+\frac{1}{z-\alpha_{2}}+\cdots+\frac{1}{z-\alpha_{k}}=\frac{k}{z-\alpha}
$$

lies in C." Rephrased in the language of Gaussian attractions, it asserts that when unit masses are placed at $k$ points $\alpha_{1}, \cdots, \alpha_{k}$ lying in a circle and the entire mass $k$ is then concentrated at a single point $\alpha$ so as to produce the same resultant force at a given exterior point $z$, the point $\alpha$ must lie somewhere in the circle.

I have cited this second theorem of Walsh because, in considering a very different matter, I have been led to the same equation but with $z$ as the unknown in place of $\alpha$. If a polynomial equation $P_{n}(z)=0$ and its first derived equation are both transformed by the same entire linear substitution $z=a z^{\prime}+b$, they pass over into a new equation and its first derived equation. This is not the case when a fractional linear substitution

$$
z=\frac{a z^{\prime}+b}{c z^{\prime}+d}, \quad(c \neq 0),
$$

\footnotetext{
* Curtiss [78a] points out that the theorem is a "result which Laguerre expressed in homogeneous coordinates."
} 
is applied. Nevertheless, various theorems regarding the location of the roots of a polynomial and its derivative may continue to hold in the transformed region with respect to the transformed polynomial and its derivative. Consider, for instance, a theorem less specific than the above GaussLucas Theorem, according to which a circle or half-plane which contains all the roots of $P_{n}(z)$ also contains all the roots of its derivative. Apply now the transformation (10). In place of $P_{n}(z)$ we get the new polynomial

$$
Q_{n}\left(z^{\prime}\right) \equiv\left(c z^{\prime}+d\right)^{n} P_{n}\left(\frac{a z^{\prime}+b}{c z^{\prime}+d}\right) .
$$

Suppose that the transformation converts the interior of the circle or half-plane into the interior of a new circle $C^{\prime}$. Then the new circle which necessarily contains all the roots of $Q_{n}(z)$ will contain also all the roots of $Q_{n}^{\prime}(z)$. The like conclusion fails, however, when the transformed region is the exterior of $C^{\prime}$. Similarly, if Jensen's Theorem were not invalidated by a transformation converting the half-plane into the interior of $C^{\prime}$, we would have the following conclusion: When the roots of a polynomial $Q_{n}(z)$ which do not lie on the perimeter of a given circle $C^{\prime}$ are pairs of roots of equal multiplicity symmetrically located* with respect to $C^{\prime}$, all the roots of its derivative not on the circle must lie in the circles whose diameters are the segments joining the pairs of symmetrically situated roots. It is easy to show the falsity of such a conclusion by an example to the contrary. Jensen's Theorem, however, is an immediate result of two applications of the following true statement: The roots of a real polynomial which do not lie in a half-plane bounded by a real axis lie in the Jensen circles. The corresponding statement after the above transformation is that when a polynomial $Q_{n}(z)$ has its roots located in the manner just explained, the roots of the derivative so far as they do not lie on or within

* Two symmetrically situated points are inverse points with respect to the circle. 
$C^{\prime}$ must lie in the circles whose diameters are the segments joining pairs of symmetrically situated roots of $Q_{n}(z)$, and the validity of this statement has been established by Walsh through the method of repulsive particles.

These examples suffice to show the need of some criterion for determining whether a theorem regarding the mutual location of the roots of a polynomial and its derivative will hold also for the transformed polynomial and its derivative after a transformation (10). To answer this question, suppose $P_{n}(z)$ replaced by (11) in consequence of the transformation (10). The new derived equation is

$$
\begin{aligned}
Q_{n}^{\prime}\left(z^{\prime}\right) \equiv n c\left(c z^{\prime}+d\right)^{n-1} P_{n}\left(\frac{a z^{\prime}+b}{c z^{\prime}+d}\right) & \\
& +(a d-b c) \cdot\left(c z^{\prime}+d\right)^{n-2} P_{n}^{\prime}\left(\frac{a z^{\prime}+b}{c z^{\prime}+d}\right)=0,
\end{aligned}
$$

which may be successively written

and

$$
\frac{n c\left(c z^{\prime}+d\right)}{a d-b c}+\frac{P_{n}^{\prime}(z)}{P_{n}(z)}=0,
$$

$$
-\frac{n}{z-\frac{a}{c}}+\sum \frac{1}{z-\alpha_{1}}=0, \quad(i=1, \cdots, n),
$$

where the $\alpha_{i}$ are the roots of the original polynomial $P_{n}(z)$. It thus appears that the new derived equation $Q_{n}^{\prime}\left(z^{\prime}\right)=0$ results by application of the linear transformation (10) to the polynomial equation (12). Now (12) is Walsh's equation (9) regarded as an equation of $(n-1)$ th degree in $z$ with given $\alpha_{i}$ and $\alpha$. It may be written in the form

$$
n P_{n}(z)-(z-\alpha) P_{n}^{\prime}(z)=0,
$$

where $\alpha$ denotes the point $z=a / c$ which by the transformation passes over into $z^{\prime}=\infty$. For given $\alpha$, there corresponds to each pair of polynomial equations

$$
P_{n}(z)=0, \quad Q_{n-1}(z) \equiv n P_{n}(z)-(z-\alpha) P_{n}^{\prime}(z)=0,
$$


a class of substitutions (10) with $a / c=\alpha$ which replaces them by a polynomial equation $Q_{n}\left(z^{\prime}\right)=0$ and its first derived equation. Conversely, for the latter pair, there is a class of (inverse) substitutions which replaces them by pairs $P_{n}(z)=0, Q_{n-1}(z)=0$ with given $\alpha$.

The question whether a theorem regarding the root location of $P_{n}(z)$ and its derivative is valid after the transformation for $Q_{n}(z)$ and its derivative therefore reduces to the determination whether the original theorem is valid for $P_{n}(z)$ and $Q_{n-1}(z)$ before the transformation as well as for $P_{n}(z)$ and $P_{n}^{\prime}(z)$. It will be observed that $\alpha$ may have three qualitatively different positions with respect to the interior of a circle or half-plane. It may lie without, within, or on its boundary. If this interior is to be transformed by (10) into the interior of a new circle $C^{\prime}$, the point $\alpha$ must be taken without the circle $C$ or the half-plane, since it passes over into $z^{\prime}=\infty$.

So far as I know the above relationship has not been previously pointed out. I find, however, that Gonggrÿp [99] has considered the polynomial $n P_{n}(z)-z P_{n}^{\prime}(z)$ which arises by application of $z=1 / z^{\prime}$ to $P_{n}^{\prime}(z)$. He calls it the "reduite" of $P_{n}(z)$ and gives several theorems relating to its roots.

As an example of the general interrelation just pointed out, let us take Walsh's "Theorem 2" previously cited, which says that when $z$ is exterior to a circle containing all the roots of $P_{n}(z)$, the point $\alpha=a / c$ in (9) must lie in the circle. The word circle has here the general sense of the closed interior or exterior. An equivalent statement is that when the roots of $P_{n}(z)$ are all in the circle and $a / c$ lies without, the $z$-roots of (12) must all lie in the circle. By the transformation (10) this gives the restricted Lucas Theorem that when a circle contains all the roots of a polynomial $Q_{n}\left(z^{\prime}\right)$, it contains also the roots of its derivative. Conversely, Walsh's Theorem follows from this by the inverse linear transformation.

I have not time to follow the matter further but return now to the Coincidence Theorem of Szegö. It has been already 
noted that he uses it to obtain Grace's Apolarity Theorem. His easy deduction is reversible, so that the two theorems, so very different in appearance, are equivalent. Szegö then goes on to obtain in an interesting manner a wealth of theorems. The only one of these which I shall cite is a theorem of Egerváry:

Theorem of Egerváry. Let

$$
\begin{aligned}
& A(x) \equiv a_{0}+C_{1}^{n} a_{1} z+C_{2}{ }^{n} a_{2} z^{2}+\cdots+a_{n} z^{n}=0 \\
& B(x) \equiv b_{0}+C_{1}^{n} b_{1} z+C_{2}^{n} b_{2} z^{2}+\cdots+b_{n} z^{n}=0
\end{aligned}
$$

be two equations whose roots all lie in (within) the unit circle. Then the roots of the composition-equation

$$
C(x)=a_{0} b_{0}+C_{1}^{n} a_{1} b_{1} z+\cdots+a_{n} b_{n} z^{n}
$$

all lie in (within) the same circle.

For proof [77], denote by $\beta_{1}, \beta_{2}, \cdots, \beta_{n}$ the roots of (14), and by $\xi$ any root of (15), so that

$$
a_{0} b_{0}+C_{1}^{n} a_{1} b_{1} \xi+C_{2}^{n} a_{2} b_{2} \xi^{2}+\cdots+a_{n} b_{n} \xi^{n}=0 .
$$

Then if we substitute $-\xi / z$ for $z$ in (14), the resulting equation has the roots $-\xi / \beta_{1}, \cdots,-\xi / \beta_{n}$. The condition that this equation and (13) shall be apolar is precisely the equation (16). Then by Grace's apolarity theorem some one of these roots, say $-\xi / \beta_{i}$, must lie in the unit circle when all roots of (13) so do. This is impossible if $\xi$ lies without (respectively without or on) the unit circle, since $\left|\beta_{i}\right| \leqq 1$ (respectively $\left|\beta_{i}\right|<1$ ) by hypothesis. The theorem therefore follows.

If we restrict the hypothesis further and suppose that all roots of (13) and of (14) lie on the perimeter of the unit circle, then in applying the theorem just proved, we can take at pleasure the circle to be the interior or the exterior of the unit circle. It follows that no root of (15) can lie without, respectively within; consequently the roots of the composition equation will lie on the perimeter of the unit circle when those of (13) and (14) do. 
Very similar in character are theorems given by Malo* (1895) and Schur (1914). Malo's theorem [88] says that if the roots of both of the equations

$$
\begin{gathered}
a_{0}+a_{1} z+\cdots+a_{m} z^{m}=0, \\
b_{0}+b_{1} z+\cdots+b_{n} z^{n}=0
\end{gathered}
$$

are all real and those of the former are all positive or all negative, then the roots of the composition equation

$$
a_{0} b_{0}+a_{1} b_{1} z+\cdots+a_{k} b_{k} z^{k}=0,
$$

in which $k$ denotes the smaller of the two degrees $m$ and $n$, are also real; furthermore, they are all different if $a_{0} b_{0} \neq 0$ and $n \leqq m$. Schur [90] draws the same conclusion for another composition equation:

$$
a_{0} b_{0}+1 ! a_{1} b_{1} z+2 ! a_{2} b_{2} z^{2}+\cdots+k ! a_{k} b_{k} z^{k}=0 .
$$

If now we look upon (17) as any arbitrarily given equation with real roots of like sign, we may regard $b_{0}, b_{1}, \cdots, b_{n}$ as a set of multipliers which by composition with (17) will always produce an equation (18a) with only real roots. Pólya and Schur [92] call any sequence of real multipliers with this property a factor sequence of the second kind. A sequence of real factors

$$
b_{0}, b_{1}, b_{2}, \cdots
$$

is said to be of the first kind when operating on all equations (17) with real roots $(n=1,2, \cdots)$ they produce only composition equations (18a) with real roots. Laguerre in his memoirs gave various special sequences with such properties; for instance,

$$
1, q, q^{4}, q^{9}, q^{16}, \cdots, \quad|q| \leqq 1,
$$

is a sequence of the first kind. Pólya and Schur make a most interesting study of the conditions which must be imposed upon the infinite set (19) of real numbers to furnish a se-

* Malo's theorem is extended by Jensen [72] to series. 
quence of the first or second kind. If, for example, (19) is a sequence of the first kind, all non-zero elements must form a single uninterrupted succession, finite or infinite in length, the terms of which are all of same sign or of alternating sign. The two authors obtain both an algebraic and a transcendental necessary and sufficient criterion that it shall be a factor sequence of the first or second kind respectively. According to the algebraic criterion, (19) is a factor sequence of the first kind if and only if all roots of the equations

$b_{0}+C_{1}^{n} b_{1} z+C_{2}{ }^{n} b_{2} z^{2}+\cdots+C_{n}^{n} b_{n} z^{n}=0,(n=1,2,3, \cdots)$,

are real and of like sign, while it is one of the second kind when they are merely real. By the transcendental criterion it is necessary and sufficient that the real series

$$
b_{0}+\frac{b_{1}}{1 !} z+\frac{b_{2}}{2 !} z^{2}+\frac{b_{3}}{3 !} z^{3}+\cdots
$$

should converge for all values of $z$ and represent an entire function which has only roots of the same sign and is of genre 0 except for a possible factor $e^{\gamma z}$, in which $\gamma$ has the sign opposite to that of the roots. For a factor sequence of the second kind this series must be of genre 0 or 1 except possibly for a factor $e^{-\gamma^{2} z^{2}}$ ( $\gamma$ real) and have only real roots.

Somewhat earlier Petrovitch [91] considered factor sequences (19) having the property that when operating upon real polynomials of even degree possessing only imaginary roots they produce always polynomials without real roots. A class of sequences specified by him with this property has, when somewhat restricted, the form

$$
b_{n}=\int_{c}^{d} f(t) t^{n} d t, \quad(n=1,2, \cdots),
$$

where $c$ and $d$ are arbitrary fixed real limits independent of $n$ and $f(t)$ is an arbitrary real function of invariable sign between $c$ and $d$. For if for $m=2 n$ we denote the polynomial (17) by $P_{2 m}(z)$, the composition equation will be 


$$
0=a_{0} b_{0}+a_{1} b_{1} z+\cdots+a_{n} b_{n} z^{n} \equiv \int_{c}^{l} f(t) P_{2 n}(t z) d t
$$

which can not be satisfied for any real value of $z$ inasmuch as both factors of the integrand are of invariable sign. A special set of factors is also obtained by putting $b_{n}=n !$.

\section{PART II}

An infinite series $a_{0}+a_{1} z+a_{2} z^{2}+\cdots$ convergent over the entire finite plane of $z$ is, in a certain sense, a generalization of a polynomial of the $n$th degree $a_{0}+a_{1} z+\cdots+a_{n} z^{n}$. We call it an entire function. The futility of efforts to carry over theorems regarding polynomials to entire functions without restriction upon the latter is, I suppose, well known to you. A familiar example is afforded by the Fundamental Theorem of Algebra. Though a polynomial of $n$th degree has $n$ roots, an entire function need not have any zeros at all. The familiar exponential function $e^{z}=1+z+z^{2} / 2 !+\ldots$ is an example of such an entire function. Again, take the familiar theorem that if a polynomial has only real roots, its derivative has only real roots. By contrast, $(z+1) e^{z^{2}}$ is a real entire function whose only zero $z=-1$ is real, but its derivative $\left(2 z^{2}+2 z+1\right) e^{z^{2}}$ has two imaginary zeros [132]. The question therefore arises: What sort of limitations must be imposed upon entire functions to enable us to extend to them our theorems on the location of roots of polynomials?

Investigation in this direction was inaugurated by Laguerre in the 80 's or earlier. Since then little advance has been made until recently when some very interesting extensions have been obtained, notably by Pólya. Before reporting on these I must recall to you certain results and ideas familiar to the analyst. First of all, I must mention the theorem of Hurwitz that when a sequence of polynomials $P_{n}(z),(n=1,2, \cdots)$, or analytic functions converges uniformly over a closed region, the roots of the limiting analytic function within the region are the limits of the zeros of the polynomials in the sequence. In the vicinity of a $p$-fold 
root of the limit lie exactly $p$ roots of $P_{n}(z)$ for sufficiently large values of $n$. An interesting application of this indispensable theorem is to the exponential series: in any given finite region of the plane there can lie no roots of the sum $S_{n}(z)$ of the first $n$ terms for sufficiently large values of $n$.

Next, I must mention Laguerre's concept of the genre of an entire function. According to Weierstrass's FactorTheorem every entire function can be factored into the uniformly convergent form:

where

$$
F(z)=e^{G(z)} z^{r} \prod_{n=1}^{\infty}\left(1-\frac{z}{a_{n}}\right) e^{H_{n}(z)},
$$

$$
H_{n}(z)=\frac{z}{a_{n}}+\frac{1}{2}\left(\frac{z}{a_{n}}\right)^{2}+\cdots+\frac{1}{m_{n}}\left(\frac{z}{a_{n}}\right)^{m_{n}},
$$

and where $G(z)$ is an entire function, polynomial or constant, $r$ is a non-negative integer, and $m_{n}$ is an appropriate positive integer which may increase indefinitely with $n$. When the degree $m_{n}$ is limited and $G(z)$ is a polynomial, the function is said to be of finite genre, the genre $p$ being then the highest (necessary) degree in the exponents of $e$ within or without the product sign. The entire function (20) differs in structure from a polynomial by the presence of exponential factors. Hence, as Borel has remarked, one may expect that the properties of entire functions will depart more and more from those of polynomials as we increase the degree of the exponential factor.

For $p=0$ we have

so that

$$
F(z)=C z^{r} \Pi\left(1-\frac{z}{a_{n}}\right),
$$

$$
\frac{d \log F(z)}{d z} \equiv \frac{F^{\prime}(z)}{F(z)}=\frac{r}{z}+\sum \frac{1}{z-a_{n}},
$$

where $\Pi$ and $\Sigma$ are uniformly convergent over any closed finite region of the plane not containing any roots $a_{n}$ of the entire function. Correspondingly, we have for $p=1$, 


$$
\begin{gathered}
F(z)=C_{1} e^{c z} z^{r} \Pi\left(1-\frac{z}{a_{n}}\right) e^{z / a_{n}} \\
\frac{F^{\prime}(z)}{F(z)}=c+\frac{r}{z}+\sum\left(\frac{1}{z-a_{n}}+\frac{1}{a_{n}}\right)
\end{gathered}
$$

and for $p=2$,

$$
F(z)=c e^{c^{\prime} z^{2}+c^{\prime \prime} z} z^{r} \prod\left(1-\frac{z}{a_{n}}\right) e^{z / a_{n}+(1 / 2)\left(z / a_{n}\right)^{2}},
$$$$
\text { (23) } \frac{F^{\prime}(z)}{F(z)}=c^{\prime \prime}+2 c^{\prime} z+\frac{r}{z}+\sum\left(\frac{1}{z-a_{n}}+\frac{1}{a_{n}}+\frac{z}{a_{n}^{2}}\right) \text {. }
$$

When $F(z)$ is a function of finite genre and its Weierstrassian product (20) is devoid of the plaguy exponential factor before the product sign,- - that is, when $G(z)$ is a constant-we call the function primitive or canonical.

We are ready now for the extension of polynomial theorems to entire functions. Consider first the simple theorem that when the roots of a polynomial are all real, the like is true of the derivative. The simple example already given shows that this may fail for an entire function of as low genre as 2 . In the case of the real polynomial with only real roots we locate all the $n-1$ roots of the derivative by Rolle's Theorem which puts one between each two successive roots of the given polynomial, but the argument fails in the case of a real entire function because the derivative of an entire function may have an infinite number. In the case of real entire functions of genre 0 or 1 the result is still valid. For first, it can be established that there can be no imaginary roots of $F^{\prime}(z)$, either by applying the equilibrium theorem of Gauss to the right-hand members of (21) and (22) or, what amounts to the same thing, by observing that the sign of the imaginary component of each term is opposite to $y$ in $z=x+i y$, since $c$ and the $a_{n}$ are real, so that $F^{\prime}(z)$ will not vanish unless $y=0$. Next, if we differentiate the right-hand members of (21) and (22) their terms will all be negative for a real value of $z$. Consequently $F^{\prime}(x) / F(x)$ is a monotone 
decreasing function of $x$, and can vanish but once between two successive roots of the denominator $F(x)$. Thus the roots of the derivative of a given real entire function of genre 0 or 1 are all real and alternate with those of the function itself, as in the case of a given real polynomial.

The argument just indicated fails in the case of entire functions of genre 2 because of the additional terms introduced in (23). When, however, $c^{\prime}$ is negative and $\Pi$ is a canonical product of genre 1 or 0 so that the term $\left(z / a_{n}\right)^{2}$ is lacking in $\Sigma$, the argument carries through. We are led thus to pick out the same special class of functions of genre 2 which we ran across at the close of Part I, namely, real entire functions which are of genre 0 or 1 , except for an exponential factor $e^{c^{\prime} z^{2}}$ in which $c^{\prime}$ is negative.

I digress a moment at this point to mention a more general result that was just obtained. A real entire function of genre $p$ with $q$ imaginary roots possesses a derivative of the same genre which in addition to the roots accounted for by the theorem of Rolle can have at most $p+q$ imaginary and other real roots. A rigorous proof of this theorem of Laguerre is given by Borel [132].

I pass next to the consideration of another noteworthy case in which the derivative of the real entire function $F(z)$ possesses only real zeros which alternate with those of $F(z)$ on either side of the origin. It is that in which $F(z)$ is a canonical function, limited by a restriction placed upon it which at first sight appears very curious, viz., that it shall have no zero at the origin. The result is discussed in Vivanti's 1907 report on entire functions but without attributing it to any particular person. I find it established in a paper [133] of Cesaro (1884) not mentioned in Vivanti's bibliography, Cesaro's exceedingly simple proof is as follows: For every zero of the derivative of such a canonical $F(z)$ of genre $p$ we have

$$
0=\frac{F^{\prime}(z)}{F(z)}=\sum_{i}\left(\frac{1}{z-\alpha_{i}}+\frac{1}{\alpha_{i}}+\frac{z}{\alpha_{i}{ }^{2}}+\cdots+\frac{z^{p-1}}{\alpha_{i}{ }^{p}}\right),
$$


which may be written

$$
0=z^{p} \sum_{i} \frac{1}{\alpha_{i}^{p}\left(z-\alpha_{i}\right)} .
$$

Now when all the roots $\alpha_{i}$ of $F(z)$ are real and the genre $p$ is an even integer, the coefficient of $i$ in every term of $\Sigma$ in (24) has a sign opposite to that of $y$. Therefore the equation can not be satisfied for an imaginary value of $z$. On the other hand, if $p$ is an odd integer, we may put the equation into the form

$$
0=z^{p} \sum_{i} \frac{\alpha_{i} z}{\alpha_{i}^{p+1}\left(z-\alpha_{i}\right)}
$$

and then the same conclusion follows, inasmuch as for real $\alpha$ the imaginary part of the coefficient of $\alpha z /(z-\alpha)$ is

$$
-\frac{i \alpha^{2} y}{(x-\alpha)^{2}+y^{2}},
$$

which again is opposite in sign to $y$. In consequence, $F^{\prime}(z)$ has no imaginary roots. Furthermore, the derivative of each term under the summation sign in (24) for even $p$ and in (25) for odd $p$ is negative for real $z \neq \alpha_{i}$. In each case $\Sigma$ is therefore a monotone non-increasing function and consequently between any two roots $\alpha_{i}$ of $F(z)$ on the same side of the origin there lies one and only one of its derivative.

It must not be thought that the conclusions obtained concerning the reality of roots of the derivative will carry over to higher derivatives, for the suppositions underlying the proof regarding the given entire function ordinarily do not hold regarding the derivative. If, for instance, the given function is canonical, the derived function is not necessarily so. Again, a function of genre $p$ has a derivative of genre $p$ at most, and an example has been given of a function of genre $p$ with a derivative of genre $p-1$. The question therefore arises: What are then the necessary and sufficient conditions to be imposed upon a given entire function possessing only real roots in order that its derivatives of all 
orders shall likewise have only real roots? This very difficult question has recently been tackled by Pólya.

The nature of the answer seems to have been suggested by the following theorem.

THEOREM OF LAGUERRE. ${ }^{*}$ If a sequence of polynomials whose roots are all real converges over every finite region of the $z$-plane, $\dagger$ the limit of the sequence is an entire function of genre 0 or 1, (by the above theorem of Hurwitz, possessing only real zeros), or the same multiplied by a factor $e^{c z^{2}}$ in which $c$ is real. Furthermore, if the roots of the polynomials are all of like sign, the limit, except for a possible factor $e^{a z}$ with real $a$, will be a function of genre 0 (possessing only roots of the same sign).

This theorem and others to be presently mentioned seem to me noteworthy in that they establish the genre of the function not on the basis of the asymptotic character of the coefficients or roots but by use of functional properties.

The derivatives of the polynomials in the theorem are of the same character as the polynomials themselves, having likewise only real roots (respectively real roots of the same sign), and also by a well known theorem on analytic functions converge uniformly over every finite region of the $z$-plane. The conclusions of the theorem can therefore be applied to succeeding derivatives of the entire function. Thus on the given hypothesis all derivatives have only real roots (respectively real roots of the same sign).

This application of Laguerre's Theorem stops short, however, of answering the question proposed. For, on the one hand, we are not sure that all real entire functions with real zeros are obtainable as limits of sequences of polynomials with real roots. On the other hand, there exist unreal entire functions possessing only real zeros. To get such a function we have merely to take in (20) an unreal $G(z)$ along with a canonical product $\Pi$ having only real zeros. Further-

* Oeuvres, vol. 1, 1882, pp. 174-177.

$\dagger$ Apparently uniform convergence is intended. 
more, the possibility that all the succeeding derivatives should have only real roots, if any, can be actually realized. The function $c^{\prime}\left(e^{i b z}-c\right)$, in which we take $b$ real and $|c|=1$, is an unreal entire function possessing an infinite periodic system of real zeros and no others, while its successive derivatives have no zeros at all. It is easy to see that it is of genre 1. Pólya cites $c^{\prime} e^{i c z}$ ( $c$ real) as a second instance, this being an unreal function which with its successive derivatives is devoid of imaginary zeros. He [120a] conjectures that with just these two exceptions all entire functions which together with their derivatives have only real zeros must be of the form

$$
c z^{r} e^{-\gamma^{2} z^{2}+d z} \prod\left(1-d_{n} z\right) e^{d_{n} z}
$$

in which all constants except $c$ are real and $\Sigma d_{n}^{2}$ is convergent. Note that we have here exactly the first kind of functions mentioned in the above Theorem of Laguerre with the added restriction that the coefficient of $z^{2}$ in the exterior exponential factor is negative or zero. This conjecture Pólya was able to prove on the supposition that the entire function is of finite genre and has only a finite number of zeros. It can then be written in the form $e^{Q(z)} P(z)$ where $P$ and $Q$ are polynomials, so that we have then a purely polynomial problem. He showed further that when all the roots of the entire function (except $z=0$ ) are of positive sign, we must take $\gamma=0$ and a non-positive $d$; accordingly the function except for such a possible exterior factor $e^{d z}$ is of genre zero. In 1923 Alander [124] extended this conclusion to any entire function of finite genre without the limitation to a finite number of zeros. This he did by showing that the function of finite genre, unless it has one of the two exceptional forms above noted, can have only a finite number of zeros, so that Pólya's conclusion is applicable.

The sufficiency of the form (26) for the reality of the roots of all the successive derivatives Pólya established neatly by setting up a sequence of polynomials 


$$
\begin{aligned}
& \phi_{n}(z)=c z^{r}\left(1-\frac{\gamma^{2} z^{2}}{n}\right)^{n} \\
& \cdot\left(1+\frac{d+d_{1}+\cdots+d_{n}}{m_{n}}\right)^{m_{n}} \prod_{\nu=1}^{n}\left(1-d_{\nu} z\right),(n=1,2, \cdots),
\end{aligned}
$$

which with proper choice of the positive integers $m_{n}$ converge uniformly to the function (26) in every finite region of the z-plane. The character of the limiting function then follows by Laguerre's Theorem given above.

It is interesting to note another closely related result of Pólya [136]. When the entire function has the form $e^{Q(z)} P(z)$ in which $Q(z)$ and $P(z)$ are polynomials, the number of imaginary roots of its $n$th derivative increases indefinitely with $n$ if $Q(z)$ is of higher degree than 2, and likewise if it is of degree 2 unless the coefficient of $z^{2}$ in $Q(z)$ is negative.

The interesting theorem of Laguerre above quoted was based upon the hypothesis of a sequence of polynomials with real roots which converged uniformly in every finite region of the plane. Pólya [120b] has notably lightened the hypothesis by showing that it is sufficient to demand that they converge uniformly in some finite region containing the origin. One thinks here of Stieltjes's Theorem that if a sequence of analytic functions converges uniformly in any given region and is bounded in absolute value over any larger region embracing the given region, then it also converges uniformly over this larger region. Pólya does not use this theorem but is able to base the extension of the region of uniform convergence directly upon the reality of the roots provided the limit is not identically zero. In a subsequent paper [130] he lightens even this latter requirement and asks only that the roots of the polynomials shall all lie within some sector having its vertex at the origin.

When the polynomials under consideration are segments $S_{n}(z)=a_{0}+a_{1} z+\cdots+a_{n} z^{n}$ of a power series, these results of Pólya follow immediately from a remarkable theorem of Jentzsch [141]. If, namely, the series has a finite radius of convergence $R>0$, every point of the circle of convergence 
is a limit point of the set of roots of the equations $S_{n}(z)=0$, $(n=1,2, \cdots)$. Suppose now that there is a sector with vertex at the origin free from the roots of $S_{n}(z)=0$. Then if the polynomial segments $S_{n}(z)$ converge in a region including the origin, it will follow that $R$ is infinite, since otherwise the theorem is violated. Later Szegö [142] has given an extension of the theorem in which the sequence is no longer limited to a polynomial sequence obtained by segmenting a power series.

Jentzsch [143] has obtained a theorem which derives the existence and genre of an entire function from suppositions regarding the roots of its segments without making the slightest demand regarding the convergence of the sequence $S_{n}(z),(n=1,2, \cdots)$. Denote by $\beta_{1, n}, \beta_{2, n}, \cdots, \beta_{n, n}$ the $n$ roots of $S_{n}(z)$. Then if from and after some fixed value of $n$ these roots satisfy the inequality

$$
\sum_{i=1}^{n} \frac{1}{\left|\beta_{i n}\right|^{\rho}}<M, \quad(n=r, r+1, \cdots),
$$

where $\rho$ is some positive integer and $M$ a fixed positive number, the series will converge over the entire plane and its limit will be an entire function of genre equal to or less than $\rho$. As a particular application of this result consider any series whose segments $S_{n}(z)$ have only real roots. By the theory of symmetric functions we have

$$
\sum_{i=1}^{n} \frac{1}{\left|\beta_{\text {in }}\right|^{2}}=\frac{a_{1}^{2}-2 a_{0} a_{2}}{a_{0}^{2}} .
$$

Here $\rho=2$, and $M$ is any number greater than the right-hand member. It follows, therefore, if the polynomial sections $S_{n}(z)$ of a formally given series have only real roots, the series converges to a limit, and the limit is an entire function of genre 2 at most. Pólya [120b] (1913) cuts down the genre to 1 at most, and to 0 when the roots are all of the same sign. Jentzsch states also, without giving proof, in his 1914 Comptes Rendus note [144], that his conclusion will hold 
also for any sequence of polynomials $a_{n 0}+a_{n 1} z+\cdots+a_{n n} z^{n}$, $(n=1,2, \cdots)$, whose roots satisfy the inequality (27), provided that it converges uniformly in some region of the plane. In the same year Lindwart and Pólya [128] considered such a sequence under the same supposition but with the added limitation (presumably unnecessary) that the region of uniform convergence shall include the origin, and proved not only that it has a limit which is an entire function of genre equal to or less than $\rho$ but that also the limit is actually a function of genre $\rho-1$ at highest except for a possible factor $e^{c z^{\rho}}$.

I turn lastly to a topic as yet but too little worked, the determination of the conditions which must be imposed upon the coefficients of an entire function to secure a specified distribution of its roots. In the case of a real polynomial of the $n$th degree, it has been long known that if its roots are distinct, the number of pairs of imaginary roots is equal to the number of changes of sign in the sequence

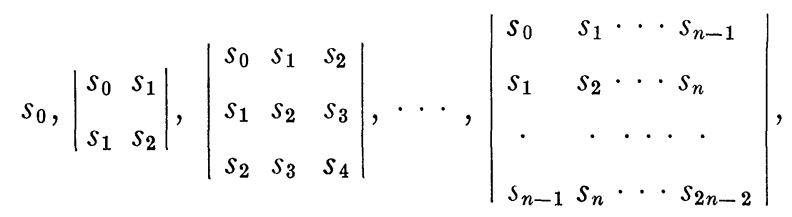

where $s_{0}=n$, and $s_{i}$ denotes the sum of the $i$ th power of the roots. The roots will be all real if and only if all of these determinants are positive. If the members of (28) are all positive when we advance every subscript by unity, we have, as Grommer [121] has shown, the necessary and sufficient condition that the roots should all be distinct and positive, provided that there is no root at the origin.

With this proviso, it is obviously permissible to replace the roots by their reciprocals, or, in other words, to substitute $s_{-i}$ for $s_{i}$. This is necessary before we can think of generalizing the condition so as to get a corresponding result for entire functions. But the indefinitely continued sequence (28) is obviously unusable even for an entire function of 
genre 0 with an infinite number of roots, for though the sum of the $i$ th powers of the reciprocals of the roots will converge for all values of $i$, the element $s_{0}=n$ increases indefinitely with the degree of the polynomial. Fortunately, in place of (28) we can use the sequence

$$
s_{-2},\left|\begin{array}{cc}
s_{-2} & s_{-3} \\
s_{-3} & s_{-4}
\end{array}\right|, \cdots,\left|\begin{array}{cccc}
s_{-2} & s_{-3} & \cdots & s_{-n-1} \\
\cdot & \cdot & \ldots & \cdot \\
s_{-n-1} & s_{-n} & \cdots & s_{-2 n}
\end{array}\right|
$$

to test the reality of the roots of a polynomial of $n$th degree. The roots are all real when and only when the terms of the sequence (29) are either all positive or are positive up to a certain member inclusive, after which they are all zero [121, p. 114]. The elements $s_{-i}$ therein contained have a significance for entire functions of genre 0 or 1 , the sum of the $i$ th powers of the reciprocals of the roots being convergent for $i \geqq 2$. According to Grommer, Hurwitz considered the question whether the roots must all be real when the terms of (29) continued indefinitely are all positive for such a function. But the elements $s_{-i}$ in (29) may be assigned a significance for any entire function. If, in fact, we take an entire function $F(z)=a_{0}+a_{1} z+\cdots$ without a zero at the origin and expand the negative of its logarithmic derivative into a series

$$
-\frac{F^{\prime}(z)}{F(z)}=s_{-1}+s_{-2} z+s_{-3} z^{2}+\cdots,
$$

the successive coefficients are connected by linear recurrence formulas in which both the $s_{-i}$ and the $a_{i}$ appear linearly. When the function is of genre 0 the $s_{-i}$ are actually equal to the sum of the $i$ th powers of the reciprocals of the roots by virtue of the relation

$$
-\frac{F^{\prime}(z)}{F(z)} \equiv-\sum_{i=1}^{\infty} \frac{1}{z-\alpha_{i}}=\sum_{i=1}^{\infty}\left(\frac{1}{\alpha_{i}}+\frac{z}{\alpha_{i}{ }^{2}}+\cdots\right) .
$$

Whether or not this be the case, let the determinant sequence (29) continued indefinitely be formed with elements taken from the series (30). With this generalization of the $s_{-i}$ 
Grommer took up the problem at Toeplitz's suggestion and proved that when the indefinitely continued sequence (29) is merely restricted by the requirement that all its terms shall be positive, the entire function will be of genre 0 or 1 except possibly for a factor $e^{-\gamma^{2} z^{2}}$ ( $\gamma$ real). His proof is complicated, involving the use of quadratic forms, continued fractions and Stieltjes integrals. Later, in 1920, Kritikos [122] under the instigation of Pólya re-derived the result by a simpler method. Grommer derives also a more general theorem, according to which the necessary and sufficient condition that a real entire function $F(z)$, for which $F(0) \neq 0$, shall have an infinite number of roots all real and simultaneously shall be an entire function of genre $2 m-1$ or less, except for a factor $e^{-\gamma^{2} z^{2 m}}$ ( $\gamma$ real), is that all the determinants

$$
\left|\begin{array}{llll}
s_{-2 m} & s_{-2 m-1} & \cdots & s_{-2 m-k+1} \\
\cdot & \cdot & \cdots & \\
s_{-2 m-k+1} & s_{-2 m-k} & \cdots & s_{-2 m-2 k+2}
\end{array}\right|,(k=1,2, \cdots),
$$

shall be positive.

It may not be without interest to say a word or two regarding the opposite possibility that all the roots shall be imaginary. Long ago I showed [2] very easily that if

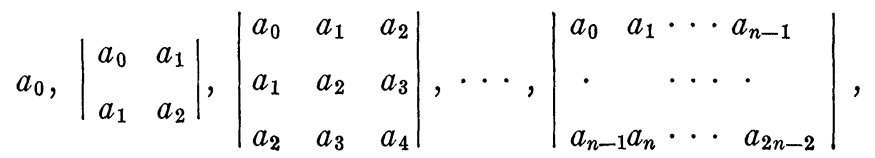

$$
\begin{aligned}
& \left(a_{i}=0 \text { for } i>n\right) \text {, }
\end{aligned}
$$

are all positive, all the roots of the polynomial

$$
P_{n}(z)=a_{0}+a_{1} z+\cdots+a_{n} z_{n}
$$

are imaginary if $n$ is even, or all but one if $n$ is odd. The condition is sufficient but not necessary. It follows that if for an entire function $a_{0}+a_{1} z+a_{2} z^{2}+\cdots$ all the terms of the sequence (31), indefinitely continued, are positive, it is an imaginary function with only imaginary zeros. A diver gent power series may also be consistent with the requirement 
of the positive character of (31), as will be seen by increasing $a_{0}, a_{2}, a_{4}$, sufficiently rapidly.

This I trust is sufficient to indicate the kind of theorems which are being obtained by extension of the theory of equations into the province of the theory of analytic functions. The mine has been yet imperfectly worked and much ore remains to be extracted.

\section{BIBLIOGRAPHY}

In the following bibliography are listed memoirs on the location of roots of polynomials and entire functions which have appeared since 1900 in the English, French, German, or Italian languages. Incidentally a very few earlier articles are included, to which reference is made in the foregoing lecture.

Work on special polynomials (Legendre's, Hermite's, Tschebyscheff's etc.) and on computation of roots or on successive approximation by iterative or other processes is excluded. It is hoped that the bibliography is sufficiently complete for the investigator or reader in the field under consideration here.

A general report on the theory of entire functions was made in 1909 by Vivanti in the following article.

1. G. Vivanti, Ueber den gegenwärtigen Stand der Theorie der ganzen transzendenten Funktionen, Archiv der Mathematik und Physik, (3), vol. 15, pp. 318-335 to which a Literaturverzeichniss with 214 entries was appended. The aspect of root location is included.

The literature on the location of roots of polynomials prior to 1900 is sufficiently covered in Epstein and Timerding's revision of Pascal's Repertorium der Hoheren Analysis, vol. I on Analysis, Chapter 4, §§15, 16.

\section{PART I. ON THE ROOTS OF POLYNOMIALS}

A. On the Number of Real or Imaginary Roots;

Number in aN INTERVAL.

2. E. B. Van Vleck, $A$ sufficient condition for the maximum number of imaginary roots of an equation of the nth degree, Annals of Mathematics, (2), vol. 4 (1903), pp. 191-192.

3. O. D. Kellogg, A necessary condition that all the roots of an algebraic equation be real, Annals of Mathematics, (2), vol. 9 (1907-8), pp. 97-98.

4. O. Dunkel, Sufficient conditions for imaginary roots of algebraic equations, Annals of Mathematics, (2), vol. 10 (1908-9), pp. 46-54.

Numbers 2-4 may be grouped together. 
5. P. Zervos, Sur les racines des équations algébriques, L'Enseignement Mathématique, vol. 6 (1904), pp. 297-299.

6. O. Nicoletti, Su alcune applicazioni del teorema di Sturm, Atti, Accademia di Torino, vol. 39 (1904), pp. 455-482.

7. G. Usai, Sopra una determinazione de funzioni di Sturm dovuta al Mollame, Giornale di Matematiche, vol. 61 (1923), pp. 77-91.

8. G. Mignosi, Teorema di Sturm e sue estensioni, Rendiconti di Palermo, vol. 49 (1925), pp. 1-164. With bibliography.

9. M. Cipolla, Il discriminante e il numero delle radici imaginarie di un'equazione algebrica a coefficienti reali, Atti, Accademia in Catania, (5), vol. 10 (1917), No. 13, pp. 1-23.

10. C. Schmidt, Ueber die obere Grenze fur die Anzahl der positiven und negativen Wurzeln einer algebraischen Gleichung, Archiv der Mathematik und Physik, vol. 15 (1909), pp. 161-164.

11. M. Fekete and G. Pólya, Ueber ein Problem von Laguerre, Rendiconti di Palermo, vol. 34 (1912), pp. 89-120.

12. A. Hurwitz, Ueber den Satz von Budan-Fourier, Mathematische Annalen, vol. 71 (1912), pp. 584-591.

13. F. Giudiche, Un'osservazione sul teorema di Budan-Fourier, Giornale di Matematiche, vol. 50 (1912), pp. 188-190.

14. D. R. Curtiss, (a) Extensions of Descartes' rules of signs connected with a problem suggested by Laguerre, Transactions of this Society, vol. 16 (1915), pp. 350-360.

(b) Recent extensions of Descartes' rule of signs, Annals of Mathematics, (2), vol. 19 (1917-18), pp. 251-278.

(c) An extension of Descartes' rule of signs, Mathematische Annalen, vol. 73 (1913), pp. 424-435.

15. G. Pólya, Ueber einige Verallgemeinerungen der Descartesschen Zeichenregel, Archiv der Mathematik und Physik, (3), vol. 23 (1915), pp. 22-32.

(Gives a new proof of Laguerre's, Runge's and Sylvester's rules of signs.)

16. N. Obrechkoff, Ueber die Trennung der reellen Wurzeln von algebraischen Gleichungen, Jahresbericht der Vereinigung, vol. 37 (1928), pp. 234-237.

17. J.v. Sz. Nagy, (a) Ueber algebraische Gleichungen mit lauter reellen Wurzeln, Jahresbericht der Vereinigung, vol. 27 (1918), pp. 37-43.

(b) Ueber geometrische Relationen zwischen den Wurzeln einer algebraischen Gleichung und ihrer Derivierten, Jahresbericht der Vereinigung, vol. 27 (1918), pp. 44-48.

(c) Zur Theorie der algebraischen Gleichungen, Jahresbericht der Vereinigung, vol. 31 (1922), pp. 238-251.

18. A. Pellet, Sur les équations ayant toutes leurs racines réelles, Comptes Rendus, vol. 147 (1908), pp. 342-343.

See also No. $88 \mathrm{a}$. 


\section{B. Upper and Lower Bounds to the Absolute Values of Roots.}

19. L. Koenigsberger, Ueber die Abgränzung der Lösungen einer algebraischen Gleichung, Rendiconti di Palermo, vol. 26 (1908), pp. 343-359.

20. S. Kakeya, On the limits of the roots of an algebraic equation with positive coefficients, Tôhoku Mathematical Journal, vol. 2 (1912-13), pp. 140-142.

(A proof that the absolute values of the roots of the polynomial $a_{n} x^{n}+a_{n-1} x^{n-1}+\cdots+a_{0}=0$ lie between the greatest and least values of the quotients $a_{n-1} / a_{n}, a_{n-2} / a_{n-1}, \cdots, a_{2} / a_{1}, a_{0} / a_{1}$.)

With this article may be connected the following five numbers:

21. T. Hayashi, (a) On a theorem of Mr. Kakeya's, Tôhoku Mathematical Journal, vol. 2 (1912-13), p. 215.

(b) On the roots of an algebraic equation, Tôhoku Mathematical Journal, vol. 3 (1913), pp. 110-115.

22. R. Kurokawa, $A$ theorem in algebra, Tôhoku Mathematical Journal, vol. 3 (1913), pp. 173-174.

23. A. Hurwitz, Ueber einen Satz des Herrn Kakeya, Tôhoku Mathematical Journal, vol. 4 (1913-14), pp. 89-93.

24. E. Landau, Abschätzung der Koeffizientensumme einer Potenzreihe, Archiv der Mathematik und Physik, (3), vol. 21 (1913), see $\$ 4$ of p. 253.

25. G. Eneström, Remarque sur un théorème relatif aux racines de l'équation $a_{n} x^{n}+\cdots+a_{0}=0$ oì tous les coefficientes sont réelles et positifs, Tôhoku Mathematical Journal, vol. 18 (1920), pp. 34-36.

(Translation of a Swedish article of 1893 in which Kakeya's Theorem was obtained.)

The following six numbers form a group:

26. R. D. Carmichael and T. E. Mason, Note on the roots of algebraic equations, this Bulletin, vol. 21 (1914), pp. 14-22.

27. G. D. Birkhoff, An elementary double inequality for the roots of an algebraic equation having greatest absolute value, this Bulletin, vol. 21 (1914), pp. 494-5.

28. K. P. Williams, Note concerning the roots of an equation, this Bulletin, vol. 28 (1922), pp. 394-6.

29. M. Kuniyeda, Note on the roots of algebraic equations, Tôhoku Mathematical Journal, vol. 9 (1916), pp. 167-173.

30. M. Fujiwara, (a) Ueber die Wurzeln der algebraischen Gleichungen, Tôhoku Mathematical Journal, vol. 8 (1915), pp. 78-85,-see part 2.

(b) Ueber die obere Schranke des absoluten Betrages der Wurzeln einer algebraischen Gleichung, Tôhoku Mathematical Journal, vol. 10 (1916), pp. 167-171.

31. R. D. Carmichael, Elementary inequalities for the roots of an algebraic equation, this Bulletin, vol. 24 (1917-18), pp. 286-296.

32. T. Kojima, (a) On a theorem of Hadamard's and its application, Tôhoku Mathematical Journal, vol. 5 (1914), see p. 58. 
(b) On the limits of the roots of an algebraic equation, Tôhoku Mathematical Journal, vol. 11 (1917), pp. 119-127.

33. M. Petrovitch, Théorème sur le nombre de racines d'une équation algébrique comprises a l'intérieur d'une circonférence donnée, Comptes Rendus, vol. 129 (1899), pp. 583 and 873.

34. S. B. Kelleher, Des limites des zéros d'un polynome, Journal de Mathématiques, (7), vol. 2 (1916), pp. 169-171.

35. S. Kakeya, On algebraic equations having the roots of limited magnitude, Proceedings of the Physico-Mathematical Society of Japan, (3), vol. 3 (1921).

36. J. L. Walsh, An inequality for the roots of an algebraic equation, Annals of Mathematics, (2), vol. 25 (1924), pp. 285-6.

\section{Regional Locations For the Roots.}

The following four numbers relate to circular regions:

37. M. Petrovitch, (a) Sur une suite de fonctions rationnelles rattachées aux équations algébriques, Bulletin de la Société Mathématique, vol. 36 (1908), pp. 141-150.

(b) Comptes Rendus, vol. 129 (1899), pp. 583 and 873.

(Considers number of roots under a given modulus.)

38. J. Schur, Journal für Mathematik, vol. 148 (1918); see \$13, pp. 134-136, Ueber Polynome, die nur im Innerm des Einheitskreis verschwinden.

(This gives a criterion that all the roots of a polynomial shall lie within the unit circle.)

39. A. Cohn, Ueber die Anzahl der Wurzeln einer algebraischen Gleichung in einem Kreise, Mathematische Zeitschrift, vol. 14 (1922), pp. 110-148.

40. G. Herglotz, Ueber die Wurzelanzahl algebraischer Gleichungen innerhalb und auf dem Einheitskreis, Mathematische Zeitschrift, vol. 19 (1924), pp. 26-34.

Nos. 41-47 relate to the equation of Hurwitz.

41. A. Hurwitz, Ueber die Bedingungen, unter welchen eine Gleichung nur Wurzeln mit negativen reellen Theilen besitzt, Mathematische Annalen, vol. 46 (1895), pp. 273-284.

42. E. Bompiani, Sulle condizioni sotto le quali un'equazione a coefficientr reali ammette solo radici con parte reale negativa, Giornale di Matematiche, vol. 49, (1911), pp. 33-39.

43. G. Andreoli, Sui limiti superiori dei moduli delle radici complesse di una data equazione algebrica, Napoli Rendiconti (3), vol. 19, (1913), pp. 97-105.

44. Liénard and Chipart, Sur le signe de la partie réelle des racines d'une Equation algébrique, Journal de Mathématiques, (6), vol. 10 (1914), pp. 291-346. Comptes Rendus, vol. 157 (1913), pp. 691-694 and 837-840.

45. L. Orlando, (a) Sui problema di Hurwitz relativo alle parti reali delle radici di un'equazione algebrica, Mathematische Annalen, vol. 71 (1912), pp. 233-245. 
(b) Sopra alcuni polinomii definiti, considerati da Hurwitz, Atti, Accademia dei Lincei, Rendiconti, Classe di scienze fisiche, matematiche e naturali, (5), vol. 22 (1913), pp. 213-215.

(c) The same, vol. 19, (1910), pp. 801-805, vol. 192 (1910), pp. 317-321 and 430-435, and vol. 20 (1911), pp. 742-745.

46. I. Schur, Ueber algebraische Gleichungen, die nur Wurzeln mit negativen Realteilen besitzen, Zeitschrift für angewandte Mathematik und Mechanik, vol. 1 (1921), pp. 307-311.

47. M. Fujiwara, Ueber die algebraischen Gleichungen, deren Wurzeln in einem Kreise oder in einer Halbebene liegen, Mathematische Zeitschrift, vol. 24 (1926), pp. 160-169.

48. See part 1 of number 30(a) above.

49. A. J. Kempner, Ueber die Separation komplexer Wurzeln algebraischer Gleichungen, Mathematische Annalen, vol. 85 (1922), pp. 49-59.

(His separation of roots is based on the arguments of the coefficients of the equation.)

50. M. Tajima, On the roots of an algebraic equation, Tôhoku Mathematical Journal, vol. 19 (1921), pp. 173-4.

51. J. L. Walsh, On Pellet's theorem concerning the roots of a polynomial, Annals of Mathematics, (2), vol. 26 (1924-25), p. 59-64.

\section{Restriction on Root location when only Certain Coefficients of the Polynomial are Given.}

52. E. Landau, (a) Ueber den Picardschen Satz, Vierteljahrsschrift der naturforschenden Gesellschaft in Zürich, vol. 51 (1906), pp. 252-318.

(b) Sur quelques gênéralisations du théorème de M. Picard, Annales de L'École Normale Supérieure, (3), vol. 24 (1907), pp. 179-201.

(Landau's articles initiated this line of work.)

53. R. E. Allardice, On a limit of the roots of an equation that is independent of all but two of the coefficients, this Bulletin, vol. 13 (1906-7), pp. 443-447.

54. P. Bohl, Zur Theorie der trinomischen Gleichungen, Mathematische Annalen, vol. 65 (1908), pp. 556-566.

55. G. Herglotz, Ueber die Wurzeln trinomischer Gleichungen, Leipziger Berichte, Math.-Phys. Classe, vol. 74 (1922), pp. 1-8.

56. P. Sergesco, Sur le module minimum des zêros de l'équation trinome, Comptes Rendus, vol. 181 (1925), pp. 762-763.

57. L. Fejér, (a) Ueber die Wurzel vom kleinsten absoluten Betrage einer algebraischen Gleichung, Mathematische Annalen, vol. 65 (1908), pp. 413423. Comptes Rendus, vol. 145 (1907), pp. 459-461.

(b) Ueber Kreisgebiete, in denen eine Wurzel einer algebraischen Gleichung liegt, Jahresbericht der Vereinigung, vol. 26 (1917), pp. 114-128.

58. P. Montel, Sur les modules des zéros des polynômes, Annales de l'École Normale Supérieure, (3), vol. 40 (1923), pp. 1-34.

Comptes Rendus, vol. 174 (1922), pp. 850-852, 1220-1222. 
59. J. L. Walsh, Sur un théorème d'algèbre, Comptes Rendus, vol. 176 (1923), pp. 1361-3.

60. E. B. Van Vleck, On limits to the absolute values of the roots of a polynomial, Bulletin de la Société Mathématique, vol. 53 (1925), pp. 105125.

61. M. Biernacki, (a) Sur un nouveau thêorème d'algèbre, Comptes Rendus, vol. 177 (1923), pp. 1193-1194.

(b) Sur les équations algébriques contenant des paramètres arbitraires, Thèse (1927), pp. 1-145, Extrait du Bulletin de l'Académie Polonaise des Sciences et des Lettres.

See also No. 51.

\section{E. Roots of the Derivative.}

$E_{1}$. The Gauss-Lucas Theorem and the Method of Repulsive Particles.

62. Gauss, Note appended at end of memoir (1816), Collected Works, 3 , p. 112; see also Osgood, Lehrbuch der Funktionentheorie, 2d ed. (1912), pp. 210-211.

63. F. Lucas, (a) Numerous articles in the Comptes Rendus, vols. 67, $75,78,89,106$. So far as I know, the Gauss-Lucas theorem is first enunciated in restricted form on p. 274 of vol. 78 (1874); completely in vol. 89 (1879), pp. 224-266.

(b) Géométrie des polynômes, Journal de l'École Polytechnique, cahier 46 (1879), pp. 1-33.

(This includes much of his previous works. Lucas considered repeatedly the curves obtained by equating to a constant the modulus or the argument of a polynomial. The curves were treated by Darboux and recently without references to Lucas by de la Vallée-Poussin in the following paper.)

64. Ch. J. de la Vallée Poussin, Sur les relations qui existent entre les racines d'une équation algébrique et celles de sa dérivée, Mathesis, vol. 22 (1902), supplement, pp. 1-20.

(Lucas theorem is reproved in Nos. 65-67, 137.)

65. Berlothy, Sur les équations algébriques, Comptes Rendus, vol. 99 (1884), pp. 745-747.

(Apparently in ignorance of the same proof by Lucas.)

66. F. Irwin, Relation between the roots of a rational integral function and its derivative, Annals of Mathematics, (2), vol. 16 (1914-5), p. 138.

67. T. Hayashi, Relation between the zeros of a rational integral function and its derivative, Annals of Mathematics, (2), vol. 15 (1913-4), pp. 112-113.

68. M. Bôcher, A problem in statics and its relation to certain algebraic invariants, Proceedings of the American Academy of Arts and Sciences, vol. 40 (1904), pp. 469-484.

(Bôcher's application of Gauss' method to the Jacobian of two binary forms is continued in the following memoirs of Walsh.) 
69. J. L. Walsh, On the location of the roots of the Jacobian of two binary forms, and of the derivative of a rational function, Transactions of this Society, vol. 19 (1918), pp. 291-298; also vol. 22 (1921), pp. 101-116, and vol. 24 (1926), pp. 31-69. See further pp. 87-88 of vol. 23.

70. D. R. Curtiss, $A$ mechanical analogy in the theory of equations, Vicepresidential address, Section A, American Association for the Advancement of Science, Toronto, 1921, Science, vol. 55 (1922), pp. 189-194.

(This address gives a general review of the applications of Gauss' method of repulsive particles.)

71. J. L. Walsh, (a) On the location of the roots of the derivative of a polynomial, Comptes Rendus du Congrès International des Mathématiciens, Strasbourg, 1920, pp. 339-342.

(b) On the location of the roots of the derivative of a polynomial, Proceedings of the National Academy, vol. 18 (1922), pp. 139-141.

(c) Sur la position des racines des dérivées d'un polynome, Comptes Rendus, vol. 172 (1921), pp. 662-664.

E. The Theorem of Jensen, with Additions by Walsh.

72. J. L. W. V. Jensen, Recherches sur la thêorie dés équations, Acta Mathematica, vol. 36 (1913), pp. 181-195.

(A statement of the theorem is given without proof on p. 190.)

73. J. L. Walsh, On the location of the roots of the derivative of a polynomial, Annals of Mathematics, (2), vol. 22 (1920-21), pp. 128-144.

74. W. H. Echols, Note on the roots of the derivative of a polynomial, American Mathematical Monthly, vol. 27 (1920-21), pp. 299-230.

74a. J.v. Sz. Nagy, Zur Theorie der algebraischen Gleichungen, Jahresbericht der Vereinigung, vol. 31 (1922), pp. 238-251.

E. The Theorem of Grace. Nos. 75-79.

75. J. H. Grace, The zeros of a polynomial, Proceedings of the Cambridge Philosophical Society, vol. 11 (1902), pp. 352-357.

76. P. J. Heawood, Geometrical relations between the roots of $f(x)=0$, $f^{\prime}(x)=0$, Quarterly Journal of Mathematics, vol. 38 (1907), pp. 84-107.

77. G. Szegö, Bemerkungen zu einem Satz von J. H. Grace über die Wurzeln algebraischer Gleichungen, Mathematische Zeitschrift, vol. 13 (1922), pp. 28-55.

78. J. L. Walsh, On the location of the roots of certain types of polynomials, Transactions of this Society, vol. 24 (1922), pp. 163-180.

78a. D. R. Curtiss, $A$ note on the preceding paper, Transactions of this Society, vol. 24 (1922), pp. 181-184.

79. J. Egerváry, On a maximum-minimum problem and its connection with the roots of equations, Szeged Acta, vol. 1 (1922), pp. 38-45.

See also No. 39. 


\section{E4. Roots of a Polynomial and its Derivatives. Linear Combinations}

80. S. Kakeya, On zeros of a polynomial and its derivatives, Tôhoku Mathematical Journal, vol. 11 (1917), pp. 5-16.

81. H. B. Mitchell, On the imaginary roots of a polynomial and the real roots of its derivative, Transactions of this Society, vol. 19 (1918), pp. 43-52.

82. G. Pólya, Ueber die Nullstellen sukzessiver Derivierten, Mathematische Zeitschrift, vol. 12 (1922), pp. 36-60.

83. A. Hurwitz, Ueber definite Polynome, Mathematische Annalen, vol. 73 (1913), pp. 173-176.

84. M. Fujiwara, Einige Bemerkungen über die elementare Theorie der algebraischen Gleichungen, Tôhoku Mathematical Journal, vol. 9 (1916), pp. 102-108.

85. T. Hayashi, On some algebraic equations having real roots only, Tôhoku Mathematical Journal, vol. 14 (1918), pp. 334-339.

86. Y. Uchida, (a) On the relation between the roots of $f(z)=0$ and $f^{\prime}(z)=0$, Tôhoku Mathematical Journal, vol. 10 (1916), pp. 139-141.

(b) On the roots of the algebraic equation of the form $f+k_{1} f^{\prime}+\cdots$ $+k_{n} f^{(n)}=0$, Tôhoku Mathematical Journal, vol. 14 (1918), pp. 325-327.

87. K. Oishi, On the roots of an algebraic equation $f+k_{1} f^{\prime}+k_{2} f^{\prime \prime}+\cdots$ $+k_{n} f^{(n)}=0$, Tôhoku Mathematical Journal, vol. 20 (1922), pp. 1-17.

See also Nos. 72, 93.

\section{F. Roots of Connected Polynomials.}

Nos. 88-92 form a group.

88. E. Malo, Note sur les équations algébriques dont toutes les racines sont reélles, Journal de Mathématiques Spéciales, (4), vol. 4 (1895), pp. 7 etc.

88 a. N. Obrechkoff, Sur un problème de Laguerre, Comptes Rendus, vol. 177 (1923), pp. 102-103. (Gives an extension of Malo's theorem.)

89. G. Szegö, see No. 77.

90. J. Schur, Zwei Sätze über algebraische Gleichungen mit lauter reellen Wurzeln, Journal für Mathematik, vol. 144 (1914), pp. 75-88.

91. M. Petrovitch, Équations algébriques et transcendantes dépourvues de racines réelles, Bulletin de la Société Mathématique, vol. 41 (1913), pp. 194-206.

92. Pólya and Schur, Ueber zwei Arten von Faktorenfolgen in der Theorie der algebraischen Gleichungen, Journal für Mathematik, vol. 144 (1914), pp. 89-113.

93. M. Fujiwara, Ueber definite polynome, Tôhoku Mathematical Journal, vol. 6 (1914), pp. 20-26.

94. S. Kakeya, On some positive forms, Tôhoku Mathematical Journal, vol. 6 (1914-15), pp. 27-31.

95. J. L. Walsh, Note on the location of the roots of a polynomial, Mathematische Zeitschrift, vol. 24 (1926), pp. 733-742.

Nos. 96-98 relate to the theorem of Biehler (Journal für Mathematik, vol. 87, p. 350 . 
96. A. Auric, Généralisation d'un théorème de Laguerre, Comptes Rendus, vol. 137 (1903), pp. 967-969.

97. M. Fujiwara, see No. 84, p. 105.

98. Y. Okada, On some algebraic equations whose roots are real and distinct, Tôhoku Mathematical Journal, vol. 14 (1918), pp. 328-333.

99. B. Gonggrÿp, Quelques théorèmes concernant la relation entre les zéros d'un polynôme et ceux d'un polynôme de degré inférieur, Journal de Mathématiques, (7), vol. 1 (1915), pp. 353-365.

100. O. Szász, Ueber Hermitesche Formen mit rekurrierender Determinante und uber rationale P.lynome, Mathematische Zeitschrift, vol. 11 (1921), pp. 23-57.

101. C. F. Gummer, The relative distribution of the real roots of a system of polynomials, Transactions of this Society, vol. 23 (1922), pp. 265-282.

102. J. v. Sz. Nagy, Ueber die Lage der Wurzeln von linearen Verknüpfungen algebraischer Gleichungen, Szeged Acta, vol. 1 (1923), pp. 127-138.

103. M. Fekete, Beweis eines Satzes von Jentzsch, Jahresbericht der Vereinigung, vol. 31 (1922), Nachtrag, pp. 42-48.

\section{G. Miscellaneous.}

$\mathrm{G}_{1}$. Analogs to the Theorem of Rolle, etc.

104. M. Fekete, (a) Ueber Zwischenwerte bei komplexen Polynomen, Szeged Acta, vol. 1 (1923), pp. 98-100.

(b) Analoga zu den Satzen von Rolle und Bolzano fur komplexe Polynome und Potenzreihen mit Luicken, Jahresbericht der Vereinigung, vol. 32 (1924), pp. 299-306; also 36 (1927), pp. 216-222.

(c) Ueber Gebiete, in denen komplexe Polynome jeden Wert zwischen zwei gegebenen annehmen, Mathematische Zeitschrift, vol. 22 (1925), pp. 1-7.

(d) Ueber die Nullstellenverteilung bei Polynomen, deren Wert an zwei Stellen gegeben ist, Jahresbericht der Vereinigung, vol. 34 (1926), pp. 220-233.

105. E. Bálint, Bemerkung zur der Note des Herrn Fekete, Jahresbericht der Vereinigung, vol. 34 (1925), pp. 233-237.

106. J. v. Sz. Nagy, Ueber einen Satz von M. Fekete, Jahresbericht der Vereinigung, vol. 32 (1924), pp. 307-309.

$\mathrm{G}_{2}$. Roots of the Characteristic Equation. Nos. 107-110.

107. G. Pick, Ueber die Wurzeln der charakteristischen Gleichungen von Schwingungsproblemen, Zeitschrift für angewandte Mathematik und Mechanik, vol. 2 (1922), pp. 353-357.

108. I. Bendixson, Sur les racines d'une équation fondamentale, Acta Mathematica, vol. 25 (1902), pp. 359-365.

109. A. Hirsch, Sur les racines d'une équation fondamentale, Acta Mathematica, vol. 25 (1902), pp. 367-370.

110. T. J. I'a Bromwich, (a) On the roots of the characteristic equation of a linear substitution, Acta Mathematica, vol. 30 (1906), pp. 297-304.

(b) Same subject, British Association of Science, 1904, pp. 440-441. 
$\mathrm{G}_{3}$. Cerrtain Minimum-Polynomials. Nos. 111-113.

111. L. Fejér, (a) Ueber die Lage der Nullstellen von Polynomen, die aus Minimumforderungen gewisser Art entspringen, Mathematische Annalen, vol. 85 (1922), pp. 41-48.

112. M. Fekete and J. L. v. Neumann, Ueber die Lage der Nullstellen gewisser Minimumpolynome, Jahresbericht der Vereinigung, vol. 31 (1922), pp. $125-138$.

113. N. Obrechkoff, Ueber die Wurzeln von algebraischen Gleichungen, Jahresbericht der Vereinigung, vol. 33 (1924), pp. 52-64.

\section{PART II. ON THE ROOTS OF ENTIRE FUNCTIONS}

H. Upper and Lower Limits to the Absolute Value of the Roots.

114. M. Petrovitch, (a) Remarque sur les zéros des séries de Taylor, Bulletin de la Société Mathématique, vol. 29 (1901), pp. 303-312.

(b) Remarque sur les zéros des fonctions entières, Bulletin de la Société Mathématique, vol. 32 (1904), pp. 65-67.

115. E. Landau, (a) Sur quelques théorèmes de M. Petrovitch relatifs aux zéros des fonctions analytiques, Bulletin de la Société Mathématique, vol. 33 (1905), pp. 251-261.

(b) Ueber eine A ufgabe aus der Funktionentheorie, Tôhoku Mathematical Journal, vol. 5 (1914), pp. 97-116.

116. S. Kakeya, On the zero points of a power series with positive coefficients, Tôhoku Mathematical Journal, vol. 3 (1913), pp. 23-24.

\section{Roots Real, or Real and of Same Sign.}

117. G. H. Hardy, On the zeros of a class of integral functions, Messenger of Mathematics, vol. 34 (1905), pp. 97-101.

118. M. Petrovitch, (a) Sur une classe de séries entières, Comptes Rendus, vol. 143 (1906), pp. 208-210.

(b) Théorème sur les séries de Taylor, Comptes Rendus, vol. 146 (1908), pp. 272-274.

(c) Une classe remarquable de séries entières, Atti del IV Congresso Internazionale dei Matematici, vol. 2 (Rome, 1908), pp. 36-43.

119. J. I. Hutchinson, On a remarkable class of entire functions, Transactions of this Society, vol. 25 (1923), pp. 324-332.

(This article connects with Nos. 117 and 118.)

120. G. Pólya, (a) Bemerkung zur Theorie der ganzen Funktionen, Jahresbericht der Vereinigung, vol. 24 (1915), pp. 392-400.

(b) Ueber Annäherung durch Polynome mit lauter reellen Wurzeln, Rendiconti di Palermo, vol. 36 (1913), pp. 279-295.

(c) Algebraische Untersuchungen über ganze Funktionen vom Geschlechte Null und Eins, Journal für Mathematik, vol. 145 (1915), pp. 224-249.

(See No. 132 below.) 
121. J. Grommer, Ganze transzendente Funktionen mit lauter reellen Nullstellen, Journal für Mathematik, vol. 144 (1914), pp. 114-165.

(With this Nos. 122 and 123 connect.)

122. N. Kritikos, Ueber ganze transzendente Funktionen mit reellen Nullstellen, Mathematische Annalen, vol. 81 (1920), pp. 97-118.

(Derives some of Grommer's results, No. 121, in simpler way.)

123. M. Fujiwara, Ueber die Nullstellen der ganzen Funktionen vom Geschlecht Null und Eins, Tôhoku Mathematical Journal, vol. 25 (1925), pp. 27-35.

124. M. Alander, Sur les fonctions entières qui ont tous les zéros sur une droite, Comptes Rendus, vol. 176 (1923), pp. 158-161.

125. A. Wahlund, Zum Beweise einer Beziehung zwischen den Koefficienten der ganzen Funktionen von der Höhe Null, die lauter negative oder lauter positive Nullstellen besitzen, Arkiv för Matematik, Astronomi, och Fysik, vol. 18 (1924), No. 22, pp. 1-5.

126. E. Maillet, Sur les fonctions entières et quasi entières, Journal de Mathématiques, (5), vol. 8 (1902), see pp. 349-350.

127. N. Tschebotareff, Ueber die Realität von Nullstellen ganzer transzendenter Funktionen, Mathematische Annalen, vol. 99 (1928), pp. 660-686.

See further Nos. 14(b), 32(b), 90, 92.

\section{J. Other Restrictions on the Roots.}

128. Lindwart and Pólya, Ueber einen Zusammenhang zwischen der Konvergenz von Polynomfolgen und der Verteilung ihrer Wurzeln, Rendiconti di Palermo, vol. 37 (1914), pp. 297-304.

129. Y. Okada, A theorem on power series, Tôhoku Mathematical Journal, vol. 15 (1919), pp. 278-279.

(Connecting with No. 128.)

130. G. Pólya, Ueber Annäherung durch Polynome, deren samtliche Wurzeln in einen Winkelraum fallen, Göttinger Nachrichten, Math.-Phys. Klasse, 1913, pp. 325-330.

131. Roots with negative real parts. See Grommer No. 121.

See also Nos. 91, 123.

\section{K. Roots of the Derivatives.}

132. E. Borel, Lȩ̧ons sur les Fonctions Entières (1900), see Chapter 2, Les Idées de Laguerre, pp. 24-47.

133. Cesaro, Sur les fonctions holomorphes de genre quelquonque, Comptes Rendus, vol. 99 (1884), pp. 26-27.

134. L. Desaint, Sur quelques points de la théorie des fonctions, Annales de l'Êcole Normale Supérieure, (3), vol. 14 (1897), see pp. 336-344.

135. L. Leau, Chapter 6, Racines des dérivées de certaines fonctions réelles de genre fini, Complément à un théorème da Laguerre, Annales de l'École Normale Supérieure, vol. 23 (1906), pp. 102-120. 
136. G. Pólya, Ueber die Nullstellen sukzessiver Derivierten, Mathematische Zeitschrift, vol. 12 (1922), pp. 36-60.

Comptes Rendus, vol. 158 (1914), pp. 330-333.

137. M. B. Porter, On a theorem of Lucas, Proceedings of the National Academy of Sciences, vol. 2 (1916), pp. 247-248, and pp. 335-336.

138. M. Alander, (a) Sur le déplacement des zéros des fonctions entières par leur dêrivation, Thèse, Upsala, 1914.

(b) Sur les zêros extraordinaires des dérivées des fonctions entières réelles, Arkiv för Matematik, Astronomi och Fysik, vol. 11 (1916)-17), No. 15, pp. 1-18.

(c) Sur les zếros des dérivées des fonctions rationnelles et d'autres fonctions méromorphes, Arkiv för Matematik, Astronomi och Fysik, vol. 14 (1919-20), No. 23, pp. 1-30.

(d) Sur les zéros complexes des dérivées des fonctions entières réelles, Arkiv för Matematik, Astronomi och Fysik, vol. 16 (1922), No. 10, pp. 1-19.

(e) Sur les fonctions entières non-réelles, Arkiv fơr Matematik, Astronomi och Fysik, vol. 18 (1924), No. 12, pp. 1-9.

139. M. Biernacki, Sur le déplacement des zéros des fonctions entières par leur dérivation, Comptes Rendus, vol. 175 (1922), pp. 18-20.

\section{Miscellaneous.}

140. F. Lukács, Eine Eigenschaft des Konvergenzkreises der Potenzreihen, Archiv der Mathematik und Physik, (3), vol. 23 (1915), pp. 34-35.

141. R. Jentzsch, (a) Untersuchungen zur Theorie der Folgen analytischer Funktionen, Dissertation, Berlin, 1914; and Acta Mathematica, vol. 41 (1918), pp. 219-251.

(b) Fortgesetzte Untersuchungen iber die Abschnitte von Potenzreihen, Acta Mathematica, vol. 41 (1918), pp. 253-270.

(Jentzsch proves that every point on the circle of convergence of a Taylor's power series is a limit point of roots of its segments. In No. 140 Lukacs showed that there was at least one such limit point on the circle. An extension of Jentzsch's result is given by Szegö in the following article.)

142. G. Szegö, (a) Ueber die Nullstellen von Polynomen, die in einem Kreise gleichmassig konvergieren, Sitzungsberichte der Berliner Matematischen Gesellschaft, vol. 21 (1922), pp. 59-64.

(b) Ueber die Nullstellen der Polynome einer Folge, die in einem einfach zusammenhängenden Gebiete gleichmässig konvergiert, Göttinger Nachrichten, 1922, pp. 137-143.

143. R. Jentzsch, Sur l'extension d'un thêorème de Laguerre, Comptes Rendus, vol. 158 (1914), pp. 780-782.

144. G. Pólya, Ueber das Graeffesche Verfahren, Zeitschrift für Mathematik und Physik, vol. 63 (1915), pp. 275-290.

Comptes Rendus, vol. 156 (1913), pp. 1145-1147.

See also Nos. 11, 72

The University of Wisconsin 